\title{
In-vivo Optical Measurement of Neural Activity in the Brain
}

\author{
Shin Ae Kim ${ }^{1}$ and Sang Beom Jun ${ }^{2,3 *}$ \\ ${ }^{1}$ School of Chemical \& Biomolecular Engineering, Georgia Institute of Technology, Atlanta, GA30332, United States, \\ Departments of ${ }^{2}$ Electronics Engineering, ${ }^{3}$ Brain and Cognitive Sciences, Ewha Womans University, Seoul 120-750, Korea
}

\begin{abstract}
The optical neural recording techniques are promising tools in recent years. Compared to the traditional electrophysiological recording, the optical means offer several advantages including no inclusion of electrical noise, simultaneous imaging of a large number of neurons, or selective recording from genetically-targeted neurons. Overall the optical neural recording technique comprises the intrinsic and the extrinsic optical recordings. The methods for intrinsic neural recording employ the change of optical properties in brains such as blood flow/oxygenation, cellular volume change, or refractive index change without addition of external indicators. Those properties can be detected using various optical techniques including laser Doppler flowmetry (LDF), nearinfrared (NIR) spectrometer, functional optical coherence tomography (fOCT), and surface plasmon resonance (SPR). The extrinsic monitoring techniques use fluorescence signals reflecting neuronal activity via chemical or genetic modification of the neuronal cells. Two most popular activity-dependent fluorescent probes, calcium indicators and voltage-sensitive fluorescent proteins will be examined in this review. The principles, the instrumentations and in vivo applications of those optical signal measurements are described.
\end{abstract}

Key words: optical neural recording, intrinsic optical recording, extrinsic optical recording, calcium indicators, voltage-sensitive fluorescent protein (VSFP)

\section{INTRODUCTION}

Mammalian brains process complex information including sensations, movements and complicated cognitive functions using sequences of action potentials in a large population of neurons. To process multiple signals simultaneously, brains take advantage of neural networks consisting of more than ten billion neurons connecting to one another. The individual neurons are known

Received August 26, 2013, Revised September 8, 2013,

Accepted September 8, 2013

${ }^{*}$ To whom correspondence should be addressed. TEL: 82-2-3277-3892, FAX: 82-2-3277-3494 e-mail: juns@ewha.ac.kr to have synaptic connections to hundreds or thousands of other neurons [1]. These intricate connections make it difficult to study functions of individual neurons or connections. Furthermore, some of the functional regions are located in the deep brain regions such as thalamus, hypothalamus, hippocampus and so on [2-4].

Various technologies have been introduced for neural recording from the mammalian brain in vivo. Microelectrodes including in vivo whole-cell patch clamping [5] and multichannel electrodes $[2,6,7]$ have been introduced to measure the electrophysiological properties in the brain. However, in general, the electrophysiological recording has limitations in the number of recording even with the state-of-the-art techniques. It is also vulnerable to environmental electrical noises and artifacts caused by electrical
Copyright $($ ) Experimental Neurobiology 2013. www.enjournal.org
This is an Open Access article distributed under the terms of the Creative Commons Attribution Non-Commercial License (http://creativecommons.org/licenses/by-nc/3.0) which permits unrestricted non-commercial use, distribution, and reproduction in any medium, provided the original work is properly cited. 
stimulation or movements. Moreover, most microelectrodes are unable to reliably record chronic neural activity [8-10]. Other techniques such as radioactive imaging of changes in blood flow, electroencephalography (EEG), magnetoencephalography (MEG), and thermal imaging [11] have also been applied in vivo with success, but still suffer from either limited spatial resolution and/or temporal resolution. Conventional noninvasive imaging techniques such as positron-emission tomography (PET), functional magnetic resonance imaging (fMRI) and diffuse optical tomography (DOT) offer three dimensional localization of active regions in the functioning human brain [12-17] without artifacts nor accessible difficulties. Despite the noninvasiveness of the functional imagings, those offer low temporal resolution and raise the issues related to the huge size and the high price of the system. For these reasons, there has been a growing need for high spatiotemporal imaging technique for the functional imaging of brain activity especially from individual neurons with miniaturized and inexpensive detection systems.

Fiber-optic technique allows minimally invasive imaging from the cortex to the deep-brain. The fibers typically include a transparent core surrounded by a transparent cladding material with a lower index of refraction and the difference of the index induces the total internal reflection. The total internal reflection causes the fibers to act as a light-guide to transmit light between the two separate ends of the fiber. Use of the fibers for in vivo brain imaging offers key mechanical benefits regarding device size, portability, and flexibility, as well as advantages concerning the performance and availability of fiber optic components [18-20].

Here, we briefly review the representative fiber optic techniques for in vivo neural signal imaging (or recording) in brain research. We first introduce four techniques for detecting intrinsic neural signals which come from the change of their nature optical properties. We also explore the techniques for extrinsic brain imaging techniques, the major spotlighted methods on neuroscience in recent years. Both intrinsic and extrinsic imaging techniques shall inform imaging modalities by use of optical fibers as well as various mechanisms for in vivo brain imaging

\section{INTRINSIC OPTICAL SIGNAL (IOS) IMAGING}

Intrinsic optical signal (IOS) imaging is a technique for measuring physiological changes associated with neuronal activity without labeling tools [21]. The labeling is unnecessary because the IOS is derived from the changes of the optical properties of neural tissues. The IOS is detected by small alterations of reflected light in the blood flow, the oxygenation of hemoglobin, the cellular volume, and the membrane potential from physiological changes

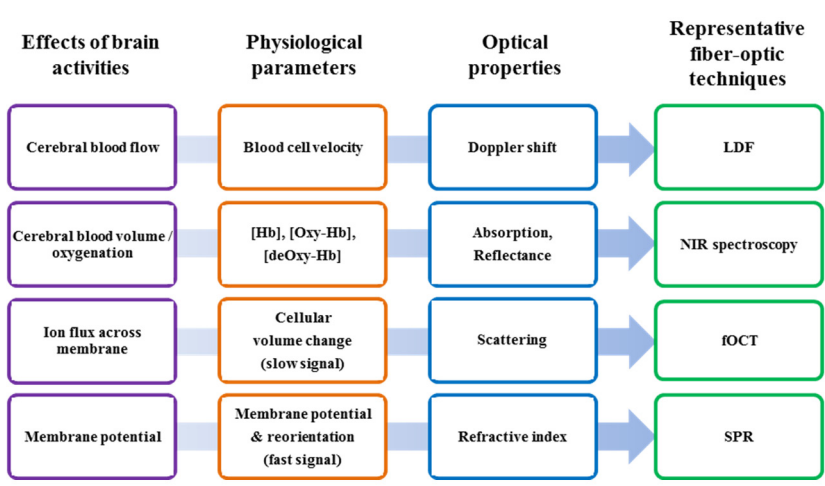

Fig. 1. Detection methods for intrinsic optical signal recording [18, 36, $37,75,76]$. The diagram illustrates the relationship between brain activity and optical measurements based on intrinsic optical contrast. The term physiological parameter is used to indicate a physiological quantity which is measured by its correspondence to an optical parameter. [Hb] indicates the concentration of hemoglobin and cytochrome-c-oxidase, respectively.

in the brain $[18,22-26]$. To measure small IOS changes from the brain, various fiber-optic techniques are employed such as laser Doppler flowmetry (LDF), near-infrared (NIR) spectrometer, functional optical coherence tomography (fOCT), and surface plasmon resonance (SPR) (Fig.1).

\section{Fiber-optic techniques for detecting IOS in brain Laser doppler flowmetry (LDF)}

Laser doppler flowmetry (LDF) is a method measuring cerebral blood flow. Cerebral blood flow correlates well with brain activity enabling for functional neuroimaging target. When low-power laser light (780 nm, 1.6 $\mathrm{mW}$ ) is emitted on the brain, the light is backscattered in dynamic red blood cells as well as in static tissues [27-29]. The light in the measuring volume of tissue is a mixture of unshifted and Doppler-shifted signal from static tissue and dynamic blood cells, respectively. The fraction of the shifted signal corresponds to the moving blood cells in the measuring region. Therefore, the magnitude and frequency distribution of the backscattered light relates to the number and velocity of moving blood cells within the measuring volume of tissue.

The first fiber-optic LDF system was presented in 1977 by Holloway and Watkins [30], and various improved versions have been successfully applied to brain imaging in various species such as rodent [27, 31], pig [32], and primate [33, 34]. In measurements of the brain function, the LDF takes advantage of the realtime continuous imaging $[27,35]$. The LDF is also non-invasive technique, not disturbing the nature physiological state of microcirculation because the LDF does not require a tight contact with target tissue. Moreover, by implementing LDF with optic fibers, it is available to access the brain without fine alignment control for 
same light-pathway between two measuring points.

\section{Near-infrared (NIR) spectroscopy}

Near-infrared (NIR) spectroscopy is performed for the noninvasive brain research because the brain is relatively transparent in the NIR range between $700 \sim 1,000 \mathrm{~nm}[19,36-38]$. The transparency comes from relatively small absorption of both water and hemoglobin within this wavelength region, so called 'optical window' The increase in the cerebral blood flow exceeding the increase in oxygen consumption leads to an increase of intravascular hemoglobin oxygenation during brain activity. The NIR light has a characteristic absorption in both oxygenated hemoglobin and deoxygenated hemoglobin [19, 36-39]. Therefore based on the NIR absorption measurements, concentration changes of oxygenated hemoglobin and deoxygenated hemoglobin can be measured during functional brain activation.

The concept that brain activity is related to intrinsic microvascular changes was asserted more than a century ago by Roy and Sherrington [40], and their assertion was demonstrated through modern imaging techniques [1, 19, 26, 36-39, 41, 42]. Jöbsis firstly demonstrated the possibility of NIR spectroscopy to detect changes of cortical oxygenation during hyperventilation on an adult brain [41]. Even though the lack of spatial resolution and attenuated NIR signal by the layering from the cortical surface remain major problems to be overcome [38, 43], the NIR images can be obtained in undemanding set-ups without invasive surgery and applicable to bedside use for human brain research. In addition, the fiber-optic NIR spectroscopy systems are commercially available. Further detail of recent NIR spectroscopy in terms of features, strengths, advantages, and limitations are reviewed in Quaresima et al. [43].

\section{Functional optical coherence tomography (fOCT)}

Optical coherence tomography (OCT) is an interferometric technique that measures scattering light from the tissue with NIR. Although standard OCT measures architectural tissue morphology, functional optical coherence tomography (fOCT) detects optical changes in cells and tissues during physiological events related to neuronal activation. The fOCT provides two-dimensional, depth-integrated activation maps of brain activity by imaging the scattering changes during the neuronal activation $[25,44$, 45]. The scattering changes contain two types of characterized signals: (1) a fast signal (less than a millisecond) from the refractive index changes at neuronal membranes by ionic composition changes; (2) a slow signal (few seconds) from the geometry changes of neuronal compartments or organelles by osmotic volume changes $[25,36]$. Both types of signals are reported with
fOCT during neuronal activation in a brain.

fOCT can be used in noninvasive imaging with high spatiotemporal resolution. Because fOCT implements NIR as its light source, large penetration depth of NIR allows noninvasive imaging of neural activity in the brain. In addition, fOCT has better spatial resolution for detecting scattered signals than NIR spectroscopy with high sensitivity to small optical changes [46]. Combination with other technologies such as polarization sensitive OCT (PSOCT) and multi-functional OCT (MF-OCT) has been providing more various information on tissue properties than standard OCT imaging alone.

\section{Surface plasmon resonance (SPR)}

A surface plasmon resonance (SPR) sensor has been also demonstrated as a recording tool of neural activity-dependent signals through the experimental approaches $[18,24,47]$ as well as numerical approaches $[48,49]$. SPR sensors have a high sensitivity about the refractive index change on a thin metal layer because they employ surface electromagnetic evanescent waves at the metaldielectric interface. The SPR signal results from the integrated refractive index changes within a very small measurement volume and the refractive index can be altered by a change in the ionic composition and/or fractional volume change between different organelles. Although the SPR mechanism underlying these fast intrinsic optical responses is not clearly understood yet, it is proven that the optical SPR signals were originated from neural activities evoked by stimulation with simultaneous electrical recording and pharmacological analysis $[18,24]$.

SPR technique was also demonstrated for in vivo detection of rat brain activity using optical fibers [18]. The fiber-optic SPR system for in vivo neural recording takes an advantage of localized high sensitivity with larger evanescent waves near the fiber probe. Further, this sensitivity will be more enhanced by using tapered optic fibers because the surface plasmon waves on the sharpened metallic surface are generated by linearly polarized incident light propagating in the core to enhance detection sensitivity [50]. Because the optical fiber is flexible and light, fiber-optic SPR system can be portable with high sensitivity for in vivo neural recording. Even though SPR requires tight contact with target cells due to the shallow penetration depth (405 nm with $635 \mathrm{~nm}$ laser), it is sufficient for localized brain functional study within a small region because of its high sensitivity on shallow regions.

\section{Applications and limits of IOS recording}

Activity-related intrinsic changes associated with neural activities or metabolisms have been used for cortical functional mapping since the 1980s. Aside from previous four imaging techniques 
various IOS imaging techniques have been developed (e.g., functional photoacoustic microscopy, NIR spectrometry, diffuse optical tomography, etc.) and the popularity of IOS imaging is increased together with technical advancement.

IOS offers distinct advantages over extrinsic signal imaging. By not requiring any chemicals or virus insertion for labeling, IOS imaging is ideally suited to study chronic preparations and clinical applications. While high-level long-term expression of fluorescent probes can lead to photo-toxicity [51], IOS address the problems by measuring the natural physiology of the brain activities. Moreover, IOS imaging techniques do not require the removal of chemicals or micro-beads after imaging from the brain, and they are thereby clinically applicable [29]. Therefore, IOS imaging for intraoperative mapping in humans provides an unparalleled opportunity to examine the basic physiology and organization of the functioning human brain. The opportunity exists to study questions that are impossible in other species, such as language organization and higher cognitive functions. However, integrated signal over the measuring volume is a limit of IOS. Because the reflected signals are averaged over the volume, IOS does not allow specific target cell detection without precise manipulation. Also, since IOS is low in signal to noise ratio, higher sensitive systems are required for better interpretation of the brain activity.

\section{EXTRINSIC OPTICAL SIGNAL (EOS) IMAGING}

In recent years, using the activity-dependent fluorescence proteins, such as calcium indicators and voltage-sensitive fluorescence proteins, the extrinsic optical recording has become a versatile tool to monitor the neural activity in neuronal culture or in living animals [52]. It is mainly due to the development and the improvement of the optical probes converting physiological signals into changes in fluorescence, as well as the development of the appropriate instrumentations for fluorescence detection. Combined with the genetic modification techniques, these extrinsic optical signals are becoming a powerful means to examine neuronal signaling from targeted neurons in culture, brain slice and living animal $[53,54]$. In this chapter, the basic mechanisms and the current state of the in vivo extrinsic optical monitoring will be reviewed in terms of the optical probes and the instrumentations.

\section{Optical probes}

\section{Calcium indicator}

In the nervous system, the intracellular calcium concentration plays an important role in a variety of means. In presynaptic terminals, exocytosis of neurotransmitter is triggered by calcium influx. In postsynaptic regions in dendritic spines, the calcium transient is involved in synaptic plasticity. More importantly, calcium signaling in nucleus is known to regulate the gene transcriptions [55]. At the resting state, the intracellular calcium concentration is maintained less than $100 \mathrm{nM}$ in most neurons while the concentration rises around 10 to 100 times higher during the generation of action potentials [56]. Therefore, the recording of somatic calcium signal is commonly used to monitor the action potentials of the neurons.

In order to monitor the neural activity-dependent calcium signal, there have been a number of attempts to develop appropriate optical probes expressing the intracellular calcium signals as fluorescence changes. The first calcium indicator was employing calcium-binding photoproteins such as aequorin [57]. Later, more sensitive fluorescent calcium indicators were developed using the binding reaction between a fluorescent chromophore and calcium-selective chelators like EGTA or BAPTA . Based on this mechanism, to date, several calcium indicators with different excitation spectra and different affinities to calcium have been introduced including quin-2, fura-2, fluo-3, fluo-4, Oregon Green Bapta and so on [58-61]. Since these dyes are easy to use and have large signal to nose ratio, these dyes are now widely used in neurophysiological studies.

In order to load the calcium indicators into the intracellular domain, in early days, the dyes were delivered inside the individual neurons using a sharpened microelectrode, glass patch micropipette or electroporation. These techniques are still widely used for basic studies of calcium signaling in neurons. To load the dyes into a population of neurons simultaneously, the most popular method is the use of membrane-permeable acetoxymethyl (AM) ester forms. The AM calcium dye molecules are trapped inside the cells after the hydrophobic eater residues are removed during the entry into the cytosol. As the application for in vivo studies, the simple application of AM calcium dyes to the brain tissue results in the loading region with the diameter of several hundred micrometers.

The true breakthrough of the use of calcium indicator as an optical probe for neural activity was the introduction of genetically encoded calcium indicators (GECIs). GECIs enable the measurement of the cytosolic calcium concentration from the genetically identified neurons. Among several different methods to express GECIs, the most popular one is currently the use of viral transduction using stereotaxic injection into the targeted brain areas. Among various viral vectors, lenti-, adeno- and adenoassociated viral vectors are commonly used to deliver the GECI constructs into the neurons of interest $[62,63]$. More importantly, the specific expression is achieved via the use of the specific 
promoters or the transgenic animals with the Cre recombinase driver [64]. In addition, the production of transgenic mice with GECI expression has been also attempted recently because it would facilitate the experiment procedure tremendously [65]. Even though there have been a few successes, the generation of GECI transgenic animal is not always possible for the specific types of neurons.

\section{Voltage-sensitive probe}

Voltage-sensitive probes are the fluorescence dyes which change their intensity in response to the exerted voltage changes [66]. Since lots of physiological processes including action potentials are accompanied with changes in the membrane potential, the fluorescence measurement can be used as an optical indicator if the dye molecules are properly anchored at the membrane. In principle, the voltage-sensitive fluorescent protein (VSFP) has a paired structure of hydrocarbon chains as the anchors and a hydrophilic group for alignment of the chromophore. It is believed that the chromophore shows an electrochromic mechanism during change of the electric field. Compared to calcium indicators, voltage-sensitive fluorescence proteins were first introduced more lately and highlighted due to the fast response time and the linear measurement of membrane potential.

Recently, the successful genetically encoded voltage-sensitive probes were reported based on the insertion of VSFP into voltagegated potassium or sodium channels [67]. Even though those probes were able to express fluorescence change proportional to the membrane potential, the efficiency of targeting specific membrane was not stable and the signal to noise ratio was not high enough to be detected in vivo environment. By leading researchers, a new generation of VSFPs was developed based on Förster resonance energy transfer (FRET) sensors such as Ciona intestinalis voltage-sensitive-containing phosphate (Ci-VSP), which showed the significantly improved targeting performance and the stable fluorescence dynamics responding membrane potential signaling [68]. However, there are still challenges to overcome in order to use the voltage-sensitive fluorescent protein as an in vivo optical probe. First, low-noise fluorescence signal needs to be maintained. In in vivo imaging conditions, there exist several noise sources such as mechanical noises, haemodynamic noises and crosstalk noise [54]. Second, the present in vivo VSFP imaging is limited for superficial regions of the brain due to the limitation of the detection system [69]. The deep brain structures need to be included for the VSFP imaging in future.

\section{Instrumentations for EOS imaging}

The instrumentation for the extrinsic fluorescence detection requires the light-sensing device and the appropriate light source for the excitation. Unlike in vitro conditions, however, in vivo fluorescence detection basically requires the high-sensitive and stable equipment since the accessibility to the cells emitting fluorescence signal is not easily guaranteed especially in freely moving animals. If the neurons of interests exist at the superficial cortical layers, the optical signal can be obtained using typical detecting approaches such as photodiode arrays, charged coupled detector (CCD)-based cameras, or complementary metal-oxidesemiconductor (CMOS)-based cameras [70, 71]. Imaging at a deeper region in the brain than the superficial area usually employs confocal or two-photon microscopy. These techniques are also available for long term experiments in combination with the formation of the chronic window or thinned skull preparations. However, these methods have the limitation in the imaging depth because the deeper region imaging requires the stronger excitation light that can produce photo damages in the exposed tissue. Therefore, in order to avoid this problem, the most realistic way is the endoscopic approach which requires the insertion of optical fibers or gradient refractive index (GRIN) lenses [72, 73]. Since the endoscopic method can dramatically decrease the distance between the optics and the target fluorescent proteins, the higher SNR can be achieved at the expense of the invasiveness.

Other than the methods mentioned above, various optical techniques can be applied for in vivo measurement of fluorescence signaling mostly for reduction of noise and increased sensitivity. Electron multiplying (EM)-CCDs can significantly improve the SNR via on-chip multiplication and higher cooling performance. Another approach for high sensitive detection is to use timecorrelated single photon counting (TCSPC) system [73]. The frame rate of the imaging system is also crucial especially for detection of voltage-sensitive calcium signal since the voltage transient-based fluorescence change is as fast as action potentials unlike calcium indicators. In general applications of VSFP, the minimal frame rates are approximately $100 \sim 1,000 \mathrm{~Hz}$ while the imaging calcium transients requires less than $100 \mathrm{~Hz}$ due to the slow response time. In addition, there are increasing attempts to develop miniaturized head-mountable imaging devices for measurement in freely moving animals. For example, recently, a miniaturized system included an objective, a dichroic mirror, and a PMT [74].

\section{CONCLUSION}

Obviously, the in vivo detection of neuronal activity from the brain is essential in the study of neural circuits. Despite the widespread use of electrophysiology, the electrical recording is vulnerable to electrical noise and artifacts and has the limitation in the 
number of channels. It is also impossible to electrically record the activity exclusively from specific types of neurons. To overcome these drawbacks or to provide alternative methods, there have been a variety of studies to develop the methods for optical neural recording as briefly described in this review. In general, the intrinsic optical signal imaging is based on the measurement of various optical properties via different mechanisms. Even though these techniques have been developed for more than decades, the technical improvement is still necessary in order to be widely used for in vivo applications. On the other hands, the measurement of extrintic optical signal is more close to the practical uses in various neuroscience studies. It is partly because the fluorescence optical probes have been developed and continuously improved for long time and also because the fluorescence microscopy has been rigorously developed due to its wide applications. In addition, the most powerful aspect, the genetic encoding technique is shared with the optogenetic neuromodulation which is one of the most popular methodologies recently due to its selective modulation using light-sensitive proteins. Even though most of the optical neural recordings were shown to be feasible for in vivo measurement, there remain several challenges to overcome. It is known that the limitations of in vivo optic imaging are the low spatial resolution and the short penetration depth, due to the scattering of optical radiation in tissues. For intrinsic optical signal recordings, the higher sensitivity is required to assure the real-time measurement because the most intrinsic signal of neural activity are not detectable without averaging or post signal-processing due to their low SNR. The fiber-optic technique should be optimized for minimized insertion damage and delicate positioning of the fiber. In addition, to adopt these techniques in freely moving animals, the miniaturization of the bulky optical systems should be accompanied with the improvement of sensitivity.

\section{ACKNOWLEDGEMENTS}

This work was supported by the Smart IT Convergence System Research Center under Global Frontier Project (SIRC-20110031866), the National Research Foundation of Korea (NRF) of the Ministry of Education, Science and Technology of Korea (2011-0029485, 2012-0004003) and Ewha Global Top 5 Grant 2011 of Ewha Womans University.

\section{REFERENCES}

1. Windhorst U, Johansson H (1999) Modern techniques in neuroscience research. Springer, New York, NY.

2. Holdefer RN, Cohen BA, Greene KA (2010) Intraoperative local field recording for deep brain stimulation in Parkinson's disease and essential tremor. Mov Disord 25:2067-2075.

3. Sturm V, Lenartz D, Koulousakis A, Treuer H, Herholz K, Klein JC, Klosterkötter J (2003) The nucleus accumbens: a target for deep brain stimulation in obsessive-compulsiveand anxiety-disorders. J Chem Neuroanat 26:293-299.

4. Belmonte MK, Allen G, Beckel-Mitchener A, Boulanger LM, Carper RA, Webb SJ (2004) Autism and abnormal development of brain connectivity. J Neurosci 24:9228-9231.

5. Kodandaramaiah SB, Franzesi GT, Chow BY, Boyden ES, Forest CR (2012) Automated whole-cell patch-clamp electrophysiology of neurons in vivo. Nat Methods 9:585-587.

6. Lai HY, Liao LD, Lin CT, Hsu JH, He X, Chen YY, Chang JY, Chen HF, Tsang S, Shih YY (2012) Design, simulation and experimental validation of a novel flexible neural probe for deep brain stimulation and multichannel recording. J Neural Eng 9:036001.

7. Viventi J, Kim DH, Vigeland L, Frechette ES, Blanco JA, Kim YS, Avrin AE, Tiruvadi VR, Hwang SW, Vanleer AC, Wulsin DF, Davis K, Gelber CE, Palmer L, Van der Spiegel J, Wu J, Xiao J, Huang Y, Contreras D, Rogers JA, Litt B (2011) Flexible, foldable, actively multiplexed, high-density electrode array for mapping brain activity in vivo. Nat Neurosci 14:1599-1605.

8. Biran R, Martin DC, Tresco PA (2005) Neuronal cell loss accompanies the brain tissue response to chronically implanted silicon microelectrode arrays. Exp Neurol 195:115126.

9. Bjornsson CS, Oh SJ, Al-Kofahi YA, Lim YJ, Smith KL, Turner JN, De S, Roysam B, Shain W, Kim SJ (2006) Effects of insertion conditions on tissue strain and vascular damage during neuroprosthetic device insertion. J Neural Eng 3:196207.

10. Najafi K, Hetke JF (1990) Strength characterization of silicon microprobes in neurophysiological tissues. IEEE Trans Biomed Eng 37:474-481.

11. Shevelev IA (1998) Functional imaging of the brain by infrared radiation (thermoencephaloscopy). Prog Neurobiol 56:269-305.

12. Moonen CT, van Zijl PC, Frank JA, Le Bihan D, Becker ED (1990) Functional magnetic resonance imaging in medicine and physiology. Science 250:53-61.

13. Tootell RB, Reppas JB, Dale AM, Look RB, Sereno MI, Malach R, Brady TJ, Rosen BR (1995) Visual motion aftereffect in human cortical area MT revealed by functional magnetic resonance imaging. Nature 375:139-141.

14. Franceschini MA, Joseph DK, Huppert TJ, Diamond SG, Boas DA (2006) Diffuse optical imaging of the whole head. J 
Biomed Opt 11:054007.

15. Zeff BW, White BR, Dehghani H, Schlaggar BL, Culver JP (2007) Retinotopic mapping of adult human visual cortex with high-density diffuse optical tomography. Proc Natl Acad Sci U S A 104:12169-12174.

16. Pardo JV, Fox PT, Raichle ME (1991) Localization of a human system for sustained attention by positron emission tomography. Nature 349:61-64.

17. Benaron DA, Hintz SR, Villringer A, Boas D, Kleinschmidt A, Frahm J, Hirth C, Obrig H, van Houten JC, Kermit EL, Cheong WF, Stevenson DK (2000) Noninvasive functional imaging of human brain using light. J Cereb Blood Flow Metab 20:469-477.

18. Kim SA, Kim SJ, Moon H, Jun SB (2012) In vivo optical neural recording using fiber-based surface plasmon resonance. Opt Lett 37:614-616.

19. Mehta AD, Jung JC, Flusberg BA, Schnitzer MJ (2004) Fiber optic in vivo imaging in the mammalian nervous system. Curr Opin Neurobiol 14:617-628.

20. Flusberg BA, Cocker ED, Piyawattanametha W, Jung JC, Cheung EL, Schnitzer MJ (2005) Fiber-optic fluorescence imaging. Nat Methods 2:941-950.

21. Bahar S, Suh M, Zhao M, Schwartz TH (2006) Intrinsic optical signal imaging of neocortical seizures: the epileptic dip. Neuroreport 17:499-503.

22. Frostig RD, Lieke EE, Tso DY, Grinvald A (1990) Cortical functional architecture and local coupling between neuronal activity and the microcirculation revealed by in vivo highresolution optical imaging of intrinsic signals. Proc Natl Acad Sci U S A 87:6082-6086.

23. Malonek D, Grinvald A (1996) Interactions between electrical activity and cortical microcirculation revealed by imaging spectroscopy: implications for functional brain mapping. Science 272:551-554.

24. Kim SA, Byun KM, Lee J, Kim JH, Kim DG, Baac H, Shuler ML, Kim SJ (2008) Optical measurement of neural activity using surface plasmon resonance. Opt Lett 33:914-916.

25. Lazebnik M, Marks DL, Potgieter K, Gillette R, Boppart SA (2003) Functional optical coherence tomography for detecting neural activity through scattering changes. Opt Lett 28:1218-1220.

26. Stepnoski RA, LaPorta A, Raccuia-Behling F, Blonder GE, Slusher RE, Kleinfeld D (1991) Noninvasive detection of changes in membrane potential in cultured neurons by light scattering. Proc Natl Acad Sci U S A 88:9382-9386.

27. Dirnagl U, Kaplan B, Jacewicz M, Pulsinelli W (1989) Continuous measurement of cerebral cortical blood flow by laser-
Doppler flowmetry in a rat stroke model. J Cereb Blood Flow Metab 9:589-596.

28. Bonner R, Nossal R (1981) Model for laser Doppler measurements of blood flow in tissue. Appl Opt 20:2097-2107.

29. Swiontkowski MF (1991) Laser Doppler flowmetrydevelopment and clinical application. Iowa Orthop J 11:119126.

30. Holloway GA Jr, Watkins DW (1977) Laser Doppler measurement of cutaneous blood flow. J Invest Dermatol 69:306309.

31. Ances BM, Greenberg JH, Detre JA (1999) Laser doppler imaging of activation-flow coupling in the rat somatosensory cortex. Neuroimage 10:716-723.

32. Aadahl P, Saether OD, Stenseth R, Juul R, Myhre HO (1991) Cerebral haemodynamics during proximal aortic crossclamping. Eur J Vasc Surg 5:27-31.

33. Williams PC, Stern MD, Bowen PD, Brooks RA, Hammock MK, Bowman RL, Di Chiro G (1980) Mapping of cerebral cortical strokes in Rhesus monkeys by laser Doppler spectroscopy. Med Res Eng 13:3-5.

34. Mayevsky A, Doron A, Manor T, Meilin S, Zarchin N, Ouaknine GE (1996) Cortical spreading depression recorded from the human brain using a multiparametric monitoring system. Brain Res 740:268-274.

35. Fredriksson I, LarssonM, Strömberg T (2012) Laser Doppler flowmetry. In: Microcirculation imaging (Leahy MJ, ed), pp 67-86. Wiley-Blackwell, Weinheim.

36. Villringer A, Chance B (1997) Non-invasive optical spectroscopy and imaging of human brain function. Trends Neurosci 20:435-442.

37. Villringer A, Planck J, Hock C, Schleinkofer L, Dirnagl U (1993) Near infrared spectroscopy (NIRS): a new tool to study hemodynamic changes during activation of brain function in human adults. Neurosci Lett 154:101-104.

38. Obrig H, Villringer A (2003) Beyond the visible--imaging the human brain with light. J Cereb Blood Flow Metab 23:1-18.

39. Kety SS, Landau WM, Freygang WH, Rowland LP, Sokoloff L (1955) Estimation of regional circulation in the brain by the uptake of an inert gas. Fed Proc 14:85.

40. Roy CS, Sherrington CS (1890) On the regulation of the blood-supply of the brain. J Physiol 11:85-158.17.

41. Jöbsis FF (1977) Noninvasive, infrared monitoring of cerebral and myocardial oxygen sufficiency and circulatory parameters. Science 198:1264-1267.

42. Ferrari M, Quaresima V (2012) A brief review on the history of human functional near-infrared spectroscopy (fNIRS) development and fields of application. Neuroimage 63:921- 
935.

43. Quaresima V, Bisconti S, Ferrari M (2012) A brief review on the use of functional near-infrared spectroscopy (fNIRS) for language imaging studies in human newborns and adults. Brain Lang 121:79-89.

44. Skala MC, Tao YK, Davis AM, Izatt JA (2011) Functional optical coherence tomography in preclinical models. In: Handbook of biomedical optics (Boas DA, Pitris C, Ramanujam N, eds), pp 281-302. CRC Press, Boca Raton, FL.

45. Park BH, Pierce MC, Cense B, de Boer JF (2003) Biomedical Optics 2003, pp 179-186. International Society for Optics and Photonics, Bellingham, WA.

46. Boppart SA (2003) Optical coherence tomography: technology and applications for neuroimaging. Psychophysiology 40:529-541.

47. Zhang J, Atay T, Nurmikko AV (2009) Optical detection of brain cell activity using plasmonic gold nanoparticles. Nano Lett 9:519-524.

48. Huang Y, Xie W, Tang D, Du C (2013) Theoretical analysis of voltage-dependent fiber optic surface plasmon resonance sensor. Opt Commun 308:109-114.

49. Choi SH, Kim SJ, Im CH, Kim SA, Kim D (2011) Quantitative model for the change of optical resonance in neural activity detection systems based on surface plasmon resonance. Opt Laser Technol 43:938-948.

50. Díaz Herrera N, Esteban Ó, Navarrete MC, González-Cano A, Benito-Peña E, Orellana G (2011) Improved performance of SPR sensors by a chemical etching of tapered optical fibers. Opt Lasers Eng 49:1065-1068.

51. Packer AM, Roska B, Häusser M (2013) Targeting neurons and photons for optogenetics. Nat Neurosci 16:805-815.

52. Grienberger C, Konnerth A (2012) Imaging calcium in neurons. Neuron 73:862-885.

53. Cao G, Platisa J, Pieribone VA, Raccuglia D, Kunst M, Nitabach MN (2013) Genetically targeted optical electrophysiology in intact neural circuits. Cell 154:904-913.

54. Mutoh H, Perron A, Akemann W, Iwamoto Y, Knöpfel T (2011) Optogenetic monitoring of membrane potentials. Exp Physiol 96:13-18.

55. Lyons MR, West AE (2011) Mechanisms of specificity in neuronal activity-regulated gene transcription. Prog Neurobiol 94:259-295.

56. Berridge MJ, Lipp P, Bootman MD (2000) The versatility and universality of calcium signalling. Nat Rev Mol Cell Biol 1:1121.

57. Shimomura O, Johnson FH, Saiga Y (1962) Extraction, purification and properties of aequorin, a bioluminescent protein from the luminous hydromedusan, Aequorea. J Cell Comp Physiol 59:223-239.

58. Paredes RM, Etzler JC, Watts LT, Zheng W, Lechleiter JD (2008) Chemical calcium indicators. Methods 46:143-151.

59. Pozzan T, Arslan P, Tsien RY, Rink TJ (1982) Antiimmunoglobulin, cytoplasmic free calcium, and capping in B lymphocytes. J Cell Biol 94:335-340.

60. Tsien RY, Pozzan T, Rink TJ (1982) Calcium homeostasis in intact lymphocytes: cytoplasmic free calcium monitored with a new, intracellularly trapped fluorescent indicator. J Cell Biol 94:325-334.

61. Neher E (1995) The use of fura-2 for estimating Ca buffers and Ca fluxes. Neuropharmacology 34:1423-1442.

62. Dittgen T, Nimmerjahn A, Komai S, Licznerski P, Waters J, Margrie TW, Helmchen F, Denk W, Brecht M, Osten P (2004) Lentivirus-based genetic manipulations of cortical neurons and their optical and electrophysiological monitoring in vivo. Proc Natl Acad Sci U S A 101:18206-18211.

63. Monahan PE, Samulski RJ (2000) Adeno-associated virus vectors for gene therapy: more pros than cons? Mol Med Today 6:433-440.

64. Wirth D, Gama-Norton L, Riemer P, Sandhu U, Schucht R, Hauser H (2007) Road to precision: recombinase-based targeting technologies for genome engineering. Curr Opin Biotechnol 18:411-419.

65. Tsai PS, Friedman B, Ifarraguerri AI, Thompson BD, LevRam V, Schaffer CB, Xiong Q, Tsien RY, Squier JA, Kleinfeld D (2003) All-optical histology using ultrashort laser pulses. Neuron 39:27-41.

66. Chemla S, Chavane F (2010) Voltage-sensitive dye imaging: technique review and models. J Physiol Paris 104:40-50.

67. Siegel MS, Isacoff EY (1997) A genetically encoded optical probe of membrane voltage. Neuron 19:735-741.

68. Akemann W, Mutoh H, Perron A, Rossier J, Knöpfel T (2010) Imaging brain electric signals with genetically targeted voltage-sensitive fluorescent proteins. Nat Methods 7:643649.

69. Akemann W, Sasaki M, Mutoh H, Imamura T, Honkura N, Knöpfel T (2013) Two-photon voltage imaging using a genetically encoded voltage indicator. Sci Rep 3:2231.

70. Ross WN, Werman R (1987) Mapping calcium transients in the dendrites of Purkinje cells from the guinea-pig cerebellum in vitro. J Physiol 389:319-336.

71. Baker BJ, Kosmidis EK, Vucinic D, Falk CX, Cohen LB, Djurisic M, Zecevic D (2005) Imaging brain activity with voltage- and calcium-sensitive dyes. Cell Mol Neurobiol 25:245-282. 
72. Adelsberger H, Garaschuk O, Konnerth A (2005) Cortical calcium waves in resting newborn mice. Nat Neurosci 8:988990.

73. Cui G, Jun SB, Jin X, Pham MD, Vogel SS, Lovinger DM, Costa RM (2013) Concurrent activation of striatal direct and indirect pathways during action initiation. Nature 494:238242.

74. Sawinski J, Wallace DJ, Greenberg DS, Grossmann S, Denk W, Kerr JN (2009) Visually evoked activity in cortical cells imaged in freely moving animals. Proc Natl Acad Sci U S A
106:19557-19562.

75. Maheswari RU, Takaoka H, Kadono H, Homma R, Tanifuji M (2003) Novel functional imaging technique from brain surface with optical coherence tomography enabling visualization of depth resolved functional structure in vivo. J Neurosci Methods 124:83-92.

76. Lee J, Kim SJ (2010) Spectrum measurement of fast optical signal of neural activity in brain tissue and its theoretical origin. Neuroimage 51:713-722. 